\title{
Noise uncertainty effect on multi-channel cognitive radio networks
}

\author{
Amira Osama ${ }^{1}$, Heba A. Tag El-Dien², Ahmad A. Aziz El-Banna ${ }^{3}$, Adly S. Tag El-Dien ${ }^{4}$ \\ ${ }^{1}$ Department of Electrical and Computer Engineering, High Institutes for Engineering and Technology Al-obour, Egypt \\ ${ }^{2,3,4}$ Faculty of Engineering at Shoubra, Benha University, Egypt
}

\section{Article Info \\ Article history: \\ Revised Mar 11, 2020 \\ Keywords: \\ Cognitive radio \\ Multi-channel \\ Noise uncertainty \\ Throughput}

Received Oct 28, 2019

Accepted Mar 30, 2020

\begin{abstract}
Achieving high throughput is the most important goal of cognitive radio networks. The main process in cognitive radio is spectrum sensing that targets getting vacant channels. There are many sensing methods like matched filter, feature detection, interference temperature and energy detection which is employed in the proposed system; however, energy detection suffers from noise uncertainty. In this paper a study of throughput under noise fluctuation effect is introduced. The work in this paper proposes multi-channel system; the overall multi-channel throughput is studied under noise fluctuation effect. In addition, the proficiency of the network has been examined under different number of channels and sensing time with noise uncertainty.
\end{abstract}

Copyright $@ 2020$ Institute of Advanced Engineering and Science. All rights reserved.

\section{Corresponding Author:}

Amira Osama,

Department of Electrical and Computer Engineering,

High Institutes for Engineering and Technology Al-obour,

21 Cairo - Belbeis Desert Rd, Kaliobeya, Egypt.

Email:amiraosama111@gmail.com, amira.osama@ oi.edu.eg

\section{INTRODUCTION}

Cognitive radio (CR) using spectrum sensing technique can be used to solve the issues of spectrum underutilization [1-3]. In cognitive radio field the secondary user (SU) detect the primary user bands and depends on this decision it selects the spectrum for its communication [4-6].The CR system could be single channel or Multi-channel, choosing the system according to needs, because each system has benefits and drawbacks. In CR system, the secondary user have to detect the vacant channel [7-10]. When a vacant channel is detected, the secondary system will access that channel. However, spectrum sensing as an urgent issue in CR, demands the secondary user to powerfully and successfully sense the existence of primary signal [11-13]. Spectrum management is the core function in CR; contains various processes such as spectrum sensing (SS), spectrum decision and spectrum handoff. SS is one of the most important advantages of CR sensors network from old wireless sensor networks (WSNs). It overtakes few opticals like low SNR for primary users, time dispersion, channel fading, and noise uncertainty [14-16].

Maximum throughput of the network has been studied for several numbers of optimal CR users. Ahead of the issue of threshold mismatch of energy detectors with noise power uncertainty, a cooperative spectrum sensing method with dynamic dual threshold is expressed in [17]. In [18] the optimal sensing technique has been offered maximizing channel throughput. The offered optimal cooperative spectrum sensing (CSS) settings for wide-band sensing channels is investigated and determined specifically with few simple however dependable techniques. In [19] a new spectrum sensing adaptive algorithm considering noise uncertainty has been proposed. In [20] the researchers studied noise uncertainty effect and fading on the detection performance. In [21] researchers offered an evidence-theory based fusion rule for cooperative energy detection in existance of noise power uncertainty. 
Authors in [22] introduce a modified two-stage detection technique that relay on energy detection under noise uncertainty. Researchers in [23] examined the act of spectrum sensing, they proposed that the throughput reaches maximum at optimal sensing time. To certify how processes of primary user is not disturbed, it has to keep the spectrum-sensing capability to get vacant channel when SU needs it. Therefore, the proficiency of sensing the existence of primary signals remains important. Spectrum sensing contains several methods (e.g. Matched filter, energy detection, feature detection, interference temperature). In [24] authors studied and analyzed the proficiency of energy detector spectrum sensing method with parameters affecting its performance on Nakagami-m fading channels under noise uncertainty and without. Authors in [25] proposed a different system of two stages spectrum sensing, Adaptive two-stage spectrum sensing (ATSS), with noise uncertainty effect ATSS is a modification of a predictable two stage spectrum sensing when the decision threshold of each stage is adapted on the distance, expected noise variance and concluded noise uncertainty range. Noise uncertainty affect the performance of multi-channel is studied with different number of channel and decides which best number ti get high throughput.

\section{RESEARCH METHOD}

This paper will introduce multi-channel system which has several channels that studies how noise uncertainity affects the system performance to get the best scenario. Our proposed system here consists of muli-channel (as each SU stands for one channel in proposed system) Spectrum sensing performance has been offered to get best number of CR users [23], sensing time, Throughput R under noise fluctuation effect. The received signal for every $\mathrm{CR}$ is sampled at sampling frequency fs.

As shown in Figure 1, every cognitive frame consists of spectrum sensing time (t) and data transmission time $(\mathrm{T}-\mathrm{t})$, where $\mathrm{T}$ is the total frame time. Consider that the distribution function for noise can be summarized in an interval $\left[(1+\rho)^{-1}{\sigma_{v i}}^{2},(1+\rho) \sigma_{v i}{ }^{2}\right]$, where $\sigma_{v i}{ }^{2}$ noise variance for ith channel and $\rho$ is a parameter that quantizies the level of the uncertainity.Assuming $\mathrm{K}$ is the number of samples existing during $\mathrm{t}$. Therefore the number of samples, $\mathrm{K}=\mathrm{t} . \mathrm{fs}[23,25]$. The probability of detection and probability of false alarm for multi-channel system under noise uncertainty effect can be written as

$$
\begin{array}{ll}
P_{f}=\underset{\sigma_{v i}{ }^{2} \in\left[(1+\rho)^{-1}{\left.\sigma_{v i}{ }^{2},(1+\rho) \sigma_{v i}{ }^{2}\right]}^{\operatorname{argmax}}\right.}{\operatorname{argmax}} & \frac{1}{2} \operatorname{erfc}\left(\frac{1}{\sqrt{2}}\left(\frac{\lambda}{\sigma_{v i}{ }^{2}}-1\right) \sqrt{t} \cdot f_{s}\right) \\
P_{d}=\underset{\sigma_{v i}{ }^{2} \in\left[(1+\rho)^{-1}{\left.\sigma_{v i}{ }^{2},(1+\rho) \sigma_{v i}{ }^{2}\right]}^{\operatorname{argmax}}\right.}{\ln } \operatorname{erfc}\left(\frac{1}{\sqrt{2}}\left(\frac{\lambda}{{\sigma_{v i}{ }^{2}}^{2}}-\gamma_{i}-1\right) \sqrt{\frac{t \cdot f_{s}}{2 \gamma_{i}+1}}\right)
\end{array}
$$

Solving (1), (2) under noise we will get

$$
\begin{aligned}
& P_{f}=\frac{1}{2} \operatorname{erfc}\left(\frac{1}{\sqrt{2}}\left(\frac{\lambda}{(1+\rho) \sigma_{v i}{ }^{2}}-1\right) \sqrt{k}\right) \\
& P_{d}=\frac{1}{2} \operatorname{erfc}\left(\frac{1}{\sqrt{2}}\left(\frac{\lambda}{(1+\rho)^{-1} \sigma_{v i}{ }^{2}}-\gamma_{i}-1\right) \sqrt{\frac{k}{2 \gamma_{i}+1}}\right) \\
& P_{f}=\frac{1}{2} \operatorname{erfc}\left(\frac{1}{\sqrt{2}}\left(\frac{\lambda}{\beta \sigma_{v i}{ }^{2}}-1\right) \sqrt{k}\right) \\
& P_{d}=\frac{1}{2} \operatorname{erfc}\left(\frac{1}{\sqrt{2}}\left(\frac{\beta \lambda}{\sigma_{i}^{2}}-\frac{\gamma_{i}}{\beta}-1\right) \sqrt{\left.\frac{k}{\frac{2 \gamma_{i}+1}{\beta}}\right)}\right.
\end{aligned}
$$

where $\beta=1+\rho, \gamma_{i}$ represents the SNR at the $\mathrm{CR}$ receiver for ith channel and $\lambda$ represents the decision threshold. The throughput for the total false alarm probability in the absence of PU is [20, 23].

$$
R_{0}=\frac{T-\tau}{T} C_{o}\left(1-Q_{f t}\right) P\left(H_{o}\right)
$$

where $C_{O}$ represents the throughput in the absence of PU. The throughput for the total missed detection probability is

$$
R_{1}=\frac{T-\tau}{T} C_{1}\left(1-Q_{d t}\right) P\left(H_{1}\right)
$$


where $C_{1}$ represents the throughput in the existence of PU. From [20, 23] we get

$$
\begin{aligned}
& Q_{f t}=\left(1-P_{f}\right)^{n} \\
& Q_{d t}=\left(1-Q_{d}\right)^{n}
\end{aligned}
$$

The total throughput $\mathrm{R}$ of the CR network can be expressed from previous disscussion as

$$
R=\frac{T-t}{T}\left(C_{0}\left(1-Q_{\text {fopt }}\right) P\left(H_{0}\right)+C_{1}\left(1-Q_{\text {dopt }}\right) P\left(H_{1}\right)\right)
$$

As $\mathrm{R}$ is a function of $P_{f}$ and $P_{d}$,it will be affected by changing noise fluctuations. So the throughput will be decreased when probability of false alarm increased or probability of detection decreased. Studying $\mathrm{n}$ that represents the number of CRs in spectrum sensing. To compare this proposed system and [17] under noise fluctuation equation of the system will be

$$
P_{f}=\frac{1}{2} \operatorname{erfc}\left(\operatorname{erfc} c^{-1}\left(2 P_{d}\right) \sqrt{2 \gamma_{i} \beta+1}+\frac{m}{2} \gamma_{i} \beta\right)
$$

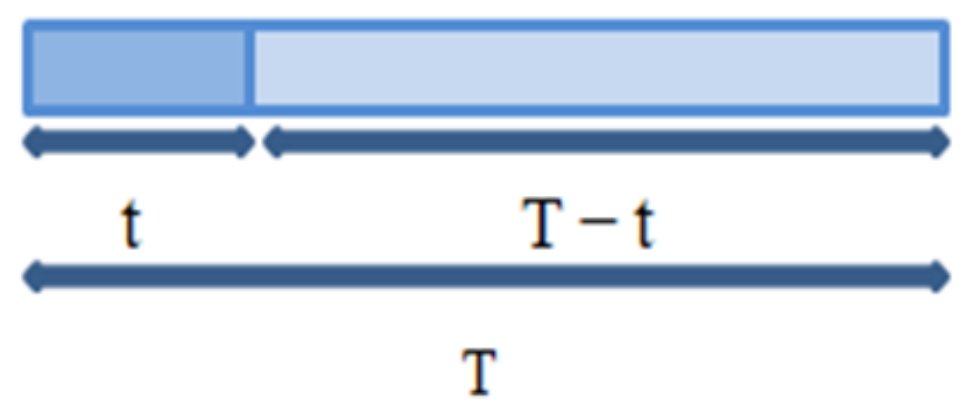

Figure 1. Cognitive frame structure

\section{RESULTS AND ANALYSIS}

Now we are going to investigate the noise fluctuation on the ED ROC. As assumed above the noise variance with uncertainty changed in the interval ${\sigma_{v i}}^{2} \boldsymbol{\epsilon}\left[{\sigma_{v i}}^{2} / \boldsymbol{\beta}, \boldsymbol{\beta}{\sigma_{v i}}^{2}\right]$ where $\beta>1$ and changed from 1.259 to 1.585 [22]. BPSK modulation has been used by primary user to transmit its data with $3 \mathrm{MHz}$ bandwidth. The maximum time for which the secondary user uninformed of the primary action is selected as Fs.T $=3000[19]$. The frame time of detection cycle is $100 \mathrm{~ms}$ and target detection probability is 0.7 . We choose $P\left(H_{0}\right)=0.8, P\left(H_{0}\right)=0.2, C_{O}=6.6582$ and $C_{O}=6.6137$. Figure 2 shows the relation between throughput and SNR at different values for noise fluctuations. We observe that when noise increasing by $30 \%$ and $\mathrm{SNR}=-12 \mathrm{~dB}$, the throughput $\mathrm{R}$ tends to zero, which means system at $\beta=1.3$ still working until SNR reaches $-12 \mathrm{~dB}$.

Figure 3 explains the throughput relation with number of CRs operating at $\mathrm{t}=2 \mathrm{~ms}$. studying at average number of $C R s=5$.At $\beta=1$, the throughput $\mathrm{R}=3.5$. At $\beta=1.05$, the throughput $\mathrm{R}=2.5$, which means by increasing $\beta$ by $5 \%$ throughput decreasing by $29 \%$. At $\beta=1.1$, the throughput $\mathrm{R}=1.8$, which means by increasing by $10 \%$ throughput decreasing by $50 \%$. At $\beta=1.3$, the throughput $\mathrm{R}=0.5$, which means by increasing by $30 \%$ throughput decreasing by $86 \%$.

Figure 4 shows the relation between throughput and sensing time with different number of CRs. (a) at $\mathrm{n}=15$, we observe that when noise increasing by $30 \%$, the throughput tends to zero. (b) at $\mathrm{n}=10$, we observe when increasing noise by $30 \%$, the throughput decreasing by $70 \%$. (c) at $n=5$, we observe when increasing noise by $30 \%$, the throughput decreasing by $60 \%$.From that we can say at $n=10$, this is the best number for CRs in this system under noise effect.

Figure 5 shows a comparison with [17] under noise fluctuation $\beta=1.3$ we obtained that at $\mathrm{t}=3 \mathrm{~ms}$, $P_{-} f$ in reference system reaches to 0.55 which means it affected from noise with $30 \%$ but in our proposed system that $P_{f}$ reaches 0.43 which means it affected from noise with $14 \%$. This means that our proposed system is better than reference system. 


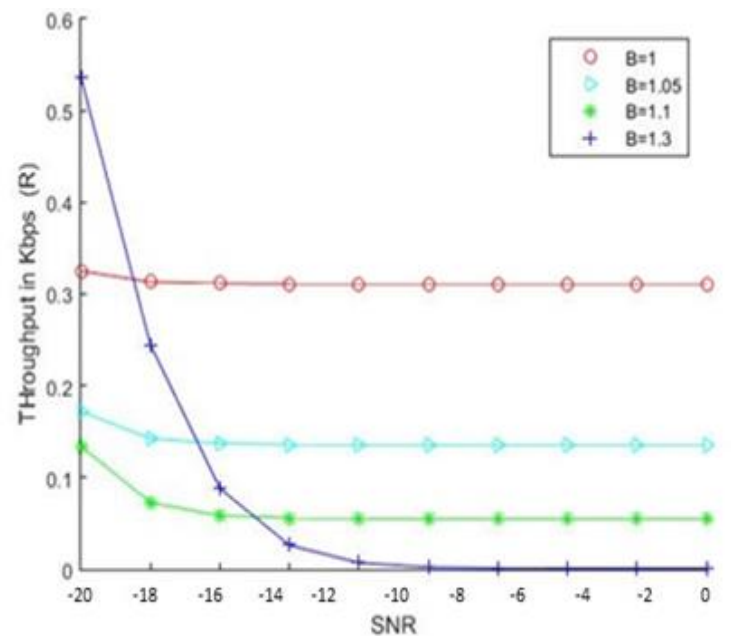

Figure 2. Throughput vs SNR

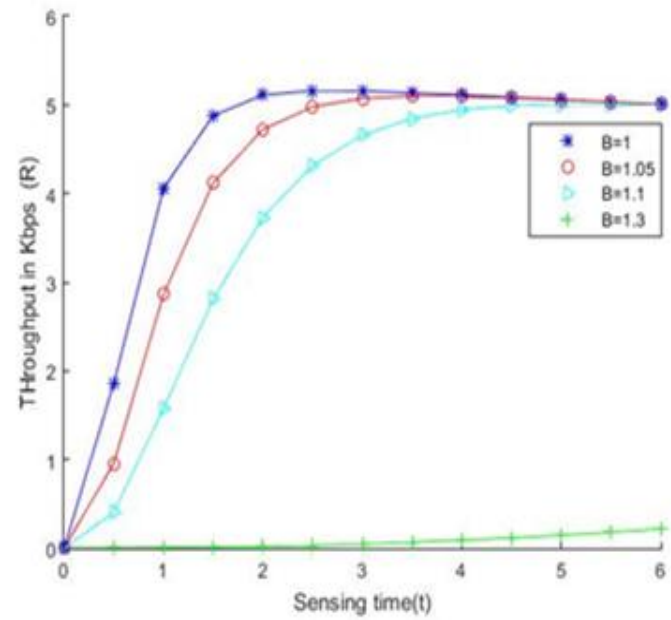

(a)
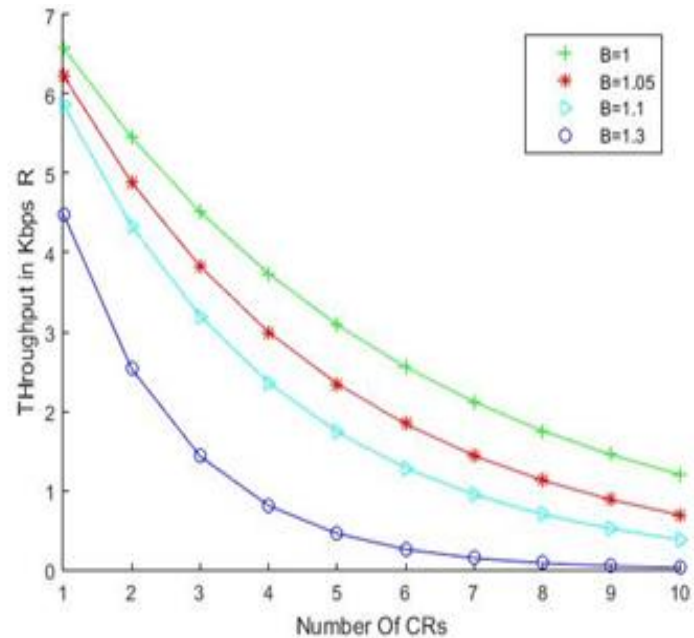

Figure 3. Throughput vs number of CRs

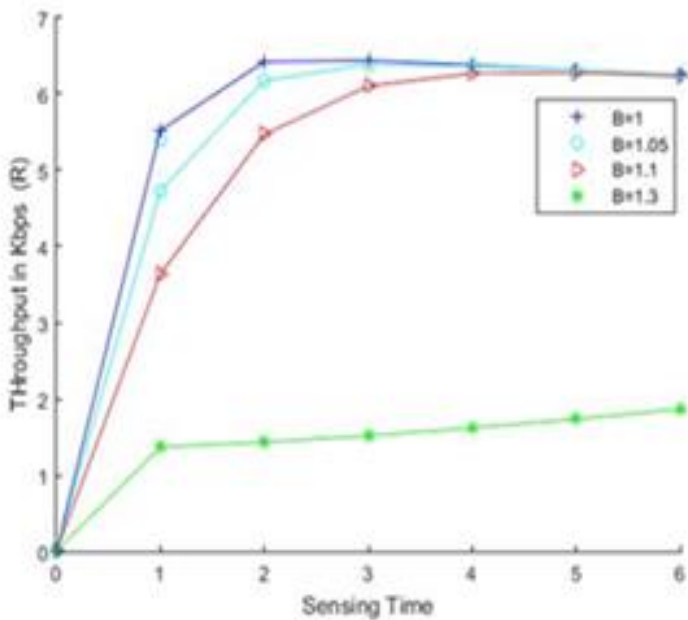

(b)

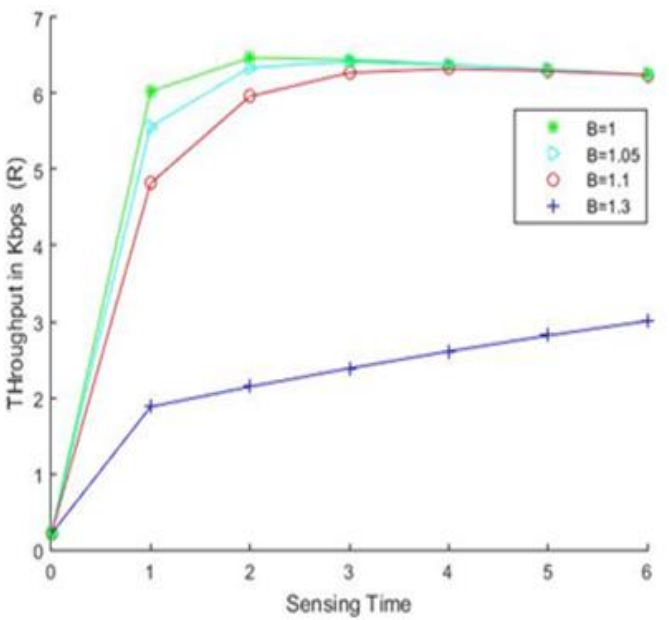

(c)

Figure 4. The relation between throughput $\mathrm{R}$ and sensing time $\mathrm{t}$, (a) No. of CRs $=15$, (b) No. of $C R s=10$, (c) No. of $C R s=5$ 


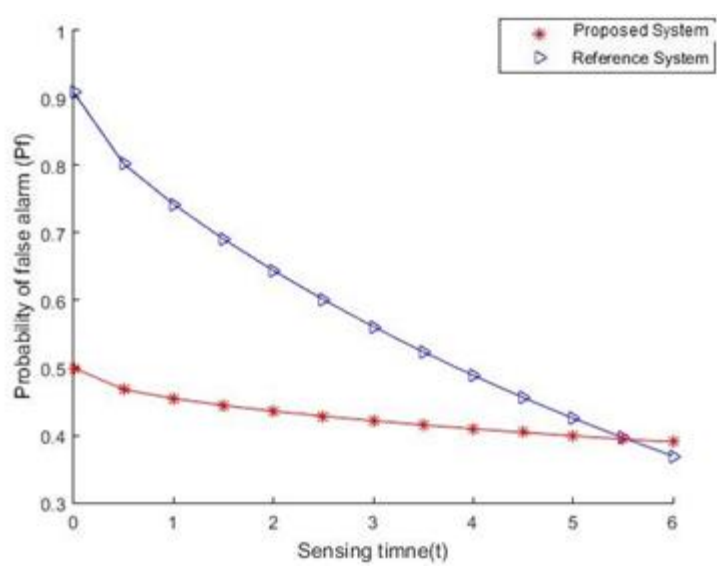

Figure 5. Sensing time vs Pf

\section{CONCLUSION}

The noise uncertainty effect on the performance of multi-channel is studied and we observe that, in multi-channel, for our system it is good to work with 10 channels to achieve reasonable throughput in relation with SNR or with sensing time by increasing noise up to $30 \%$.From that we can say ,the proposed system can reach reasonable throughput in single or multi-channel under noise that increase of $30 \%$ effect.

\section{ACKNOWLEDGEMENTS}

I would like to express my strong thanks to my supervisors for their guidance. I would like to express my Love and thank to my family members.

\section{REFERENCES}

[1] Y. Gao, et al., "Effective Capacity of Cognitive Radio Systems," 2016 IEEE $13^{\text {th }}$ International Conference on Signal Precessing (ICSP), pp. 1757-1761, 2016.

[2] D. W. Yue, et al., "Log-average-SNR ratio and cooperative spectrum sensing," IEEE Journal of Communications and Networks, vol. 18, no. 3, pp. 311-319, 2016.

[3] A. Osama, et al., "Spectrum Sensing in Single Channel and Multi-Channel Cognitive Radio Networks," Indonesian Journal of Electrical Engineering and Computer Science (IJEECS), vol. 16, no. 2, pp. 812-817, 2019.

[4] Dhivya, et al, "Ingenious Method for Conducive Handoff Appliance in Cognitive Radio Networks," International Journal of Electrical and Computer Engineering (IJECE), vol. 8, no. 6, pp. 5195-5202, 2018.

[5] S. H. Alnabelsi, et al., "Dynamic resource allocation for opportunisticsoftware-defined IoT networks: stochastic optimizationframework," International Journal of Electrical and Computer Engineering (IJECE), vol. 10, no. 4, pp. 3854-3861, 2020.

[6] R. Abdelrassoul, et al, "Comparative study of spectrum sensing for cognitive radio system using energy detection over different channels," World Symposium on Computer Applications and Research, IEEE, Cairo. 2016.

[7] M. Aljarah, et al., "Cooperative hierarchical based edge-computing approach for resources allocation of distributed mobile and IoT applications," International Journal of Electrical and Computer Engineering (IJECE), vol. 10, no. 1, pp. 296-307, 2020.

[8] H. Al-Mahdi and Y. Fouad, "Design and analysis of routing protocol for cognitive radio ad hoc networks in heterogeneous environment," International Journal of Electrical and Computer Engineering (IJECE), vol. 9, no. 1, pp. 341-351, 2019.

[9] Q. Zou, et al., "Cooperative sensing via sequential detection," IEEE Transactions on Signal Processing, vol. 58, no. 12, pp. 6266-6283, 2010.

[10] M. Subhedar and G. Birajdar, "Spectrum sensing Techniques in Cognitive Radio Networks: A survey," International Journal of Next-Generation Networks (IJNGN), vol. 3, no. 2, pp. 5277-5288, 2010.

[11] S. Chowdhury, et al., "A Throughput-efficient Cooperative Sensing and Allocation Model for Cognitive Radio Networks," 2015 IEEE International Conference on Advanced Networks and Telecommunications Systems (ANTS), pp. 1-3, 2015.

[12] A. Bhowmick, et al., "A Hybrid Cooperative Spectrum Sensing for Cognitive Radio Networks in Presence of Fading," 2015 Twenty First National Conference on Communications (NCC), Mumbai, pp. 1-6, 2015.

[13] Y. Chu et al., "Hard Decision Fusion Based Cooperative Spectrum Sensing over Nakagami-m Fading Channels," IEEE, vol. 12, no. 31, pp. 1-4. 2012.

[14] H. Li et al., "Utility-Based Cooperative Spectrum Sensing Scheduling in Cognitive Radio Networks," IEEE, pp. 1-12, 2015. 
[15] S. Zhang, et al., "Cross-layer Rethink on Sensing-throughput Tradeoff for Multi-channel Cognitive Radio Networks," IEEE Transactions on Wireless Communications, vol. 15, no. 10, pp. 6883-6897, 2016.

[16] P. Varade, et al., "Throughput maximization of cognitive radio multi relay network with interference management," International Journal of Electrical and Computer Engineering (IJECE), vol. 8, no. 4, pp. 2230-2238, 2018.

[17] R. Wan, et al., "Dynamic dual threshold cooperative spectrum sensing for cognitive radio under noise power uncertainty," Human-centic Computing and Information Science, vol. 9, no. 1, pp. 1-21, 2019.

[18] J. Shen, et al., "Maximum Channel Throughput via Cooperative Spectrum Sensing in Cognitive Radio Networks," IEEE Transactions on Wireless Communications, vol. 8, no. 10, pp. 5166-5175, 2009.

[19] D. Raman and N. P. Singh, "An Algorithm for Spectrum Sensing in Cognitive Radio under Noise Uncertainty," International Journal of Future Generation Communication and Networking, vol. 7, no. 3, pp. 61-68, 2014.

[20] Z. Quan, et al., "Optimal Multiband Joint Detection for Spectrum Sensing in Cognitive Radio Networks," IEEE Transactions on Signal Processing, vol. 57, no. 3, pp. 1128-1140, 2009.

[21] P. B. Gohain, et al., "Evidence Theory based Cooperative Energy Detection under Noise Uncertainty," GLOBECOM 2017-2017 IEEE Global Communications Conference, pp. 1-7, 2017.

[22] H. A. T. El-Dien, et al., "Noise Uncertainty Effect on a Modified Two-Stage Spectrum Sensing Technique," Indonesian Journal of Electrical Engineering and Computer Science (IJEECS), vol. 1, no. 2, pp. 341-348, 2016.

[23] A. Bhowmick, et al., "Throughput Optimization with Cooperative Spectrum Sensing in Cognitive Radio Network," IEEE International Advance Computing Conference (IACC), Gurgaon, pp. 329-332, 2014.

[24] A. Eslami and S. Karamzadeh, "Performance Analysis of Energy Based Spectrum Sensing over Nakagami-m Fading Channels with Noise uncertainty," International Journal of Electronics, Mechanical and Mechatronics Engineering, vol. 6, no. 1, pp. 1101-1106, 2016.

[25] W. Lee, et al., "Adaptive Two-stage Spectrum Sensing under Noise Uncertainty in Cognitive Radio Networks," ECTI Transactions on Electrical Engineering, Electronics, and Communications, vol. 14, no. 1, pp. 21-35, 2016.

\section{BIOGRAPHIES OF AUTHORS}
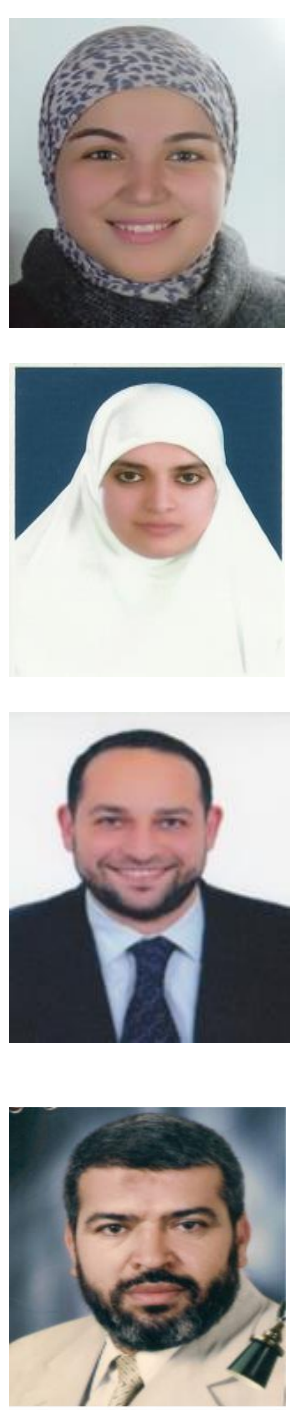

Amira Osama, Teaching Assistant in Electrical and Communication Engineering, Universitad High Institutes for Engineering and Technology Al-obour. She received her B.Eng., from Benha university (Egypt) in 2013. She has been a Teaching Assistant High Institutes for Engineering and Technology Al-obour since 2014. Her research interests include the field of network communcation, Mobile systems, WSN and IOT .

Heba A.Tag El Dien recieved B.Sc., M.Sc. and Ph.D. degrees from Shoubra Faculty of Engineering, Benha University, Egypt, in 2007, 2013,and 2017 respectively. She is currently assistant Professor in the electrical engineering department in Shoubra Faculty of Engineering. She is a legal instructor in Cisco academy in Shoubra faculty of Engineering.

Ahmad A. Aziz El-Banna received a master's degree from Benha University, Egypt, in 2011, and a Ph.D. degree from the Egypt-Japan University of Science and Technology, Egypt, in 2014. He served as a Visiting Researcher with Osaka University, Japan, from September 2013 to June 2014. He also completed a nine-month scholarship in embedded systems at Information Technology Institute, Egypt, in 2008. Since June 2018, he has been Postdoctoral Research Fellow with the Smart Sensing and Mobile Computing Laboratory, Shenzhen University, China. He also holds the position of an Assistant Professor with the Electrical Engineering Department, Faculty of Engineering at Shoubra, Benha University, Egypt. His research interests include wireless communications, embedded systems, cooperative networking, MIMO, space-time coding, IoT, WSN, underwater communication, and machine learning.

Adly S. Tag Eldien receivrd B.Sc.,M.Sc. and Ph.D. degrees Benha University, Egypt, in 1984,1989 and 1993 respectively.He is currently an associate professor in the department of electrical engineering- Benha University.He was the X-head of Benha University network and information center, his research interests include robotics, network and mobile communication. 